\title{
Finite Horizon Model Reduction of a Class of Neutrally Stable Systems with Applications to Texture Synthesis and Recognition.
}

\author{
Mario Sznaier Octavia Camps Cecilia Mazzaro \\ Department of Electrical Engineering \\ The Pennsylvania State University \\ University Park, PA 16802
}

\begin{abstract}
In this paper we address the problem of finitehorizon model reduction for a class of neutrally stable discrete-time systems. The main result of the paper shows that this problem can be solved by considering suitable defined Hankel operators and Grammians, leading to an algorithm similar to the well known balanced truncation. However, in this case the structure of the problem can be exploited to obtain tighter truncation error bounds. These results are illustrated with a non-trivial practical example arising in the context of image processing: texture synthesis and recognition.
\end{abstract}

\section{INTRODUCTION}

This paper addresses the problem of finite-horizon model reduction of discrete linear time invariant systems that have a periodic impulse response. This situation arises in the context of many practical problems from widely dissimilar areas, where the use of control-theoretic tools can result in a substantial simplification. For instance, as we will show in the sequel, solving this problem allows for developing efficient algorithms for image restoration and texture recognition. A second interesting application is obtaining low-order approximations, over a finite time horizon, of an input-output system with high-dimensional, linear Hamiltonian dynamics. This problem is at the core of explaining the apparent paradox entailed in the transition from microscopically conservative dynamics to seemingly dissipative macroscopic behavior [15], [4]

Model reduction of stable LTI systems is by now a well understood problem and a number of efficient algorithms are available (see for instance [11], [2], [7] and the textbooks [13], [20]). In comparison, relatively few results are available for the case of interest here, where the system has all its poles on the unit circle and it is only desired to find approximations over a finite time interval. Model reduction of unstable systems in the $\mathcal{L}_{\infty}$ sense has been addressed in [21]. However, contrary to the stable case, $\mathcal{L}_{\infty}$ approximation error bounds do not lead to bounds in the $\mathcal{L}_{2}[0, T]$ sense when $G$ is non Schur. Finite horizon $\mathcal{L}_{2}$ model reduction of unstable systems was addressed in [15], by recasting the problem into the reduction of an equivalent stable system obtained through $j \omega$-axis shifting techniques. While computationally attractive, this approach cannot guarantee that certain structural properties, such as periodicity of the impulse response, are preserved, a key requirement in the applications of interest here.

The present paper pursues a different approach to model reduction. Motivated by some well known results on realization theory [17], [19], [9], we work directly with the Hankel matrix of the system under consideration. Since the impulse response is periodic, this matrix is circulant and structural properties can then be exploited to obtain balanced (in a sense that we formalize in the paper) realizations in an efficient way. Moreover, directly truncating these balanced realizations provides an optimal (finite horizon) Hankel norm approximation to the original operator.

In the second portion of the paper we apply these tools to the non-trivial problems of texture synthesis and recognition. The main idea is to model images as the (periodic) impulse response of a non-necessarily causal LTI system and use the proposed method to identify the corresponding model. Partial images can be expanded and additional realizations of the same texture can be obtained by simply driving the corresponding model with a suitable input. Texture recognition can be accomplished by recasting the problem into a model (in)validation form.

\section{NOTATION}

Below we summarize the notation used in this paper: $\mathbf{x}$

$\|\mathbf{x}\|_{p} \quad$ p-norm of a vector: $\|\mathbf{x}\|_{p} \doteq$ $\left(\sum_{k=1}^{m}\left|x_{k}\right|^{p}\right)^{\frac{1}{p}}, \quad p \quad \in \quad[1, \infty)$, $\|\mathbf{x}\|_{\infty} \doteq \max _{k=1, \ldots, m}\left|x_{k}\right|$.

$\mathbf{A}^{\dagger} \quad$ Moore-Penrose pseudoinverse: $\left(\mathbf{A}^{T} \mathbf{A}\right)^{-1} \mathbf{A}^{T}$.

$\bar{\sigma}(\mathbf{A}) \quad$ maximum singular value of $\mathbf{A}$.

$\mathbf{A}>(\geq) 0 \quad \mathbf{A}=\mathbf{A}^{T}$ is positive (semi) definite.

$\mathcal{B} \mathcal{X}(\gamma) \quad$ closed $\gamma$-ball in a normed space $\mathcal{X}$ :

$\mathcal{B X}(\gamma)=\left\{x \in \mathcal{X}:\|x\|_{\mathcal{X}} \leq \gamma\right\}$

$\ell_{2}^{m} \quad$ Banach space of vector sequences equipped with the norm: 


$$
\|x\|_{2} \doteq\left(\sum_{i=0}^{\infty}\left\|\mathbf{x}_{i}\right\|_{2}^{2}\right)^{\frac{1}{2}}
$$

$\ell_{2[0, n]}^{m} \quad$ subspace of $\ell_{2}^{m}$ formed by finite sequences of length $n+1$.

$\mathcal{L}\left(\ell_{2}\right) \quad$ space of bounded operators in $\ell_{2}$.

$\mathcal{B S} \quad$ Unit ball in the space of bounded spectral density signals.

$\left\{e_{k} \in \ell_{2}: E\left(e^{j \omega}\right)^{*} E\left(e^{j \omega}\right) \leq 1\right\}$.

This paper considers finite-dimensional, discrete-time, linear shift invariant (FDLSI) operators. From an inputoutput viewpoint, such an operator $S$ can be represented by its convolution kernel $\left\{\mathbf{S}_{i}\right\}$. Causal LSI systems (i.e $\mathbf{S}_{i}=0, i<0$ ) will also be represented by a minimal state-space realization:

$$
\begin{aligned}
\mathbf{x}_{k+1} & =\mathbf{A} \mathbf{x}_{k}+\mathbf{B} u_{k} \\
y_{k} & =\mathbf{C} \mathbf{x}_{k}+\mathbf{D} u_{k} .
\end{aligned}
$$

\section{Model Reduction for a Class of Neutrally STABLE SySTEMS}

Consider a system $G_{n}$ with a state space realization of the form (1), with $\mathbf{A} \in \mathcal{R}^{n \times n}, \mathbf{B} \in \mathcal{R}^{n \times m}, \mathbf{C} \in$ $\mathcal{R}^{p \times n}$, and such that $\mathbf{A}^{n}=\mathbf{I}$. Our goal is to find an $r^{t h}$ order reduced approximation $G_{r}$ such that $\left\|G_{n}-G_{r}\right\|_{*}$ is minimized, where $*$ denotes a norm of interest, defined over the finite interval $[0, n-1]$. In addition, we will require that $\mathbf{A}_{r}^{n}=\mathbf{I}$, that is, the approximation preserves the periodicity properties of the impulse response.

Next we present an algorithm to solve this problem based on the singular value decomposition of the (finite horizon) Hankel operator of $G$.

\section{Algorithm 1:}

1.- Given the system (1), let $\mathbf{M}_{k}=\mathbf{C A}{ }^{k-1} \mathbf{B} \in \mathcal{R}^{p \times m}$ denote its $k^{\text {th }}$ Markov parameter and form the $n p \times$ $n m$ block matrix:

$$
\mathbf{H}_{n} \doteq\left[\begin{array}{cccc}
\mathbf{M}_{1} & \mathbf{M}_{2} & \ldots & \mathbf{M}_{n} \\
\mathbf{M}_{2} & \mathbf{M}_{3} & \ldots & \mathbf{M}_{1} \\
\vdots & \vdots & \ddots & \vdots \\
\mathbf{M}_{n} & \mathbf{M}_{1} & \ldots & \mathbf{M}_{n-1}
\end{array}\right]
$$

2.- Perform a singular value decomposition:

$$
\begin{array}{r}
\mathbf{H}_{n}=\left[\begin{array}{ll}
\mathbf{U} & \mathbf{U}_{\perp}
\end{array}\right]\left[\begin{array}{ll}
\mathbf{S} & \mathbf{0} \\
\mathbf{0} & \mathbf{0}
\end{array}\right]\left[\begin{array}{l}
\mathbf{V}^{T} \\
\mathbf{V}_{\perp}^{T}
\end{array}\right], \\
\mathbf{S}=\operatorname{diag}\left(\sigma_{1}, \ldots, \sigma_{n}\right), \sigma_{i} \geq \sigma_{j}, i \geq j
\end{array}
$$

3.- Assume that $\sigma_{r}>\sigma_{r+1}$ and form the reduced order model:

$$
\begin{aligned}
\mathbf{S}_{r} & =\operatorname{diag}\left(\sigma_{1}, \ldots, \sigma_{r}\right) \\
\mathbf{A}_{r} & =\mathbf{S}_{r}^{-\frac{1}{2}} \mathbf{U}_{r}^{T} \mathbf{P} \mathbf{U}_{r} \mathbf{S}_{r}^{\frac{1}{2}}, \mathbf{B}_{r}=\mathbf{S}_{r}^{\frac{1}{2}} \mathbf{V}_{r}^{(1)} \\
\mathbf{C}_{r} & =\mathbf{U}_{r}^{(1)} \mathbf{S}_{r}^{\frac{1}{2}}, \mathbf{D}_{r}=\mathbf{D}
\end{aligned}
$$

where

$$
\mathbf{P}=\left[\begin{array}{ccccc}
0 & \mathbf{I}_{p} & 0 & \ldots & 0 \\
0 & 0 & \mathbf{I}_{p} & \ldots & 0 \\
\vdots & \vdots & \ddots & \vdots & \\
\mathbf{I}_{p} & 0 & 0 & \ldots & 0
\end{array}\right]
$$

$\mathbf{U}_{r}\left(\mathbf{V}_{r}\right)$ denotes the submatrix formed by the first $r$ columns (rows) of $\mathbf{U}(\mathbf{V})$ and where $\mathbf{U}_{r}^{(1)}$ and $\mathbf{V}_{r}^{(1)}$ denote the first $p \times r$ block of $\mathbf{U}_{r}$ and $r \times m$ block of $\mathbf{V}_{r}^{T}$, respectively.

Lemma 1: The $r^{\text {th }}$ order realization (3) satisfies the structural constraint $\mathbf{A}^{n}=I$ and its corresponding $\mathrm{n}^{\text {th }}$ order Hankel operator satisfies the following truncation error bound:

$$
\left\|\mathbf{H}_{n}-\mathbf{H}_{n}^{r e d}\right\|_{*}=\left\|\mathbf{S}_{n-r}\right\|_{*}
$$

where $\mathbf{H}_{n}$ and $\mathbf{H}_{r}^{\text {red }}$ denote the $n^{\text {th }}$ order Hankel matrices corresponding to the full and reduced realizations, respectively, $\|\cdot\|_{*}$ denotes any unitarily invariant norm, and $\mathbf{S}_{n-r}=\operatorname{diag}\left(\sigma_{r+1}, \ldots, \sigma_{n}\right)$.

Proof: Given in the Appendix

Remark 1: From this Lemma it follows that, for the case under consideration here, the optimal rank- $r$ approximation to $\mathbf{H}$ obtained directly from its SVD also has a Hankel operator structure. This is in contrast to the case of general systems, where enforcing this constraint requires performing a more computationally demanding Hankel norm approximation [1], [7].

Next we show that the procedure above is equivalent to performing balanced truncations using the finite-time Grammians ${ }^{1}$.

$$
\begin{aligned}
& \mathbf{W}_{c}=\sum_{k=0}^{n-1} \mathbf{A}^{k} \mathbf{B B}^{T}\left(\mathbf{A}^{T}\right)^{k}=\mathbf{K}_{c} \mathbf{K}_{c}^{T} \\
& \mathbf{W}_{o}=\sum_{k=0}^{n-1}\left(\mathbf{A}^{T}\right)^{k} \mathbf{C}^{T} \mathbf{C} \mathbf{A}^{k}=\mathbf{K}_{o}^{T} \mathbf{K}_{o}
\end{aligned}
$$

where $\mathbf{K}_{c}$ and $\mathbf{K}_{o}$ denote Kalman's controllability and observability matrices, respectively. Due to its block-circulant structure, the Hankel matrix $\mathbf{H}_{n}$ and $\left(\mathbf{W}_{c} \mathbf{W}_{o}\right)^{\frac{1}{2}}$ have the same singular values, as when using the usual (infinitetime) definitions. The analogy is further carried by the following result:

Theorem 1: Algorithm 1 is equivalent to performing balanced truncations using the Grammian definitions (6).

Proof: Without loss of generality, consider the state space realization of the original system (1) obtained from (3) by taking $r=n$. Direct computations show that $\left(\mathbf{A}^{T}\right)^{k} \mathbf{C}^{T} \mathbf{C} \mathbf{A}^{k}=\mathbf{S}^{\frac{1}{2}}\left[\mathbf{U}^{(k)}\right]^{T} \mathbf{U}^{(k)} \mathbf{S}^{\frac{1}{2}}$, where $\mathbf{U}^{(k)}$ denotes the $k^{\text {th }}$ block of $\mathbf{U}$. Since $\mathbf{U}^{T} \mathbf{U}=\mathbf{I}$, it follows that $\mathbf{W}_{o}=\mathbf{S}^{\frac{1}{2}}\left\{\sum\left[\mathbf{U}^{(k)}\right]^{T} \mathbf{U}^{(k)}\right\} \mathbf{S}^{\frac{1}{2}}=\mathbf{S}$. A similar reasoning shows that $\mathbf{W}_{c}=\mathbf{S}$. Thus, this realization is balanced. Eliminating the states corresponding to the smallest $n-r$ singular values leads precisely to the realization (3).

Remark 2: Equation (5) for computing the approximation error is reminiscent of the well known bounds on the Hankel norm of the residual error operator obtained for balanced truncation. Note however that it is exact,

\footnotetext{
${ }^{1}$ Alternatively, these matrices can be interpreted as "average" Grammians by taking the average value $\lim _{N \rightarrow \infty} \frac{1}{N} \sum_{0}^{\infty} \mathbf{A}^{k} \mathbf{B B}^{T}\left(\mathbf{A}^{T}\right)^{k}$ and $\frac{1}{N} \sum_{k=0}^{\infty}\left(\mathbf{A}^{T}\right)^{k} \mathbf{C}^{T} \mathbf{C} \mathbf{A}^{k}$.
} 
due to the specific structure of $\mathbf{H}_{n}$, rather than an upper bound. For instance, for the case of the $\ell^{2}$ induced norm, it yields $\left\|\mathbf{H}_{n}-\mathbf{H}_{n}^{r e d}\right\|_{2}=\sigma_{r+1}$, rather than the looser upper bound $2 * \sum_{r+1}^{n} \sigma_{i}$ provided by standard balancedtruncation theory.

\section{Application: TeXture Modelling And RECOGNITION}

Texture modelling has been a long standing problem in computer vision. Statistical approaches proceed by modelling texture as a stochastic process and attempting to capture the relevant properties [5], [6]. The approach that we pursue in this paper is related to these in the sense that we will also model images exhibiting a given texture as realizations of a second order stationary stochastic process. Our starting point is to consider the intensity values $\mathcal{I}(k,:)$ of the $k^{\text {th }}$ row of the image as the output, at step $k$, of a discrete linear shift-invariant, not necessarily causal, system driven by white noise. In this context, texture modelling can be recast into the problem of identifying the relevant system model from the given images. Once these models are obtained, synthesis follows in a straightforward fashion, by simple driving the model with a suitable input. Similarly, texture recognition can be accomplished by interrogating a collection of models to determine which one is the closest to the given sample.

\section{A. Extracting models from images.}

The problem of identifying a causal, FDLSI system from samples of its output has been extensively studied in identification and several approaches, roughly divided into subspace identification [18] and operator-theoretic methods [3] are available. However, in the problem under consideration here, the unknown system is not necessarily causal: the intensity value at a pixel is likely to depend on the values of all pixels in its neighborhood, not just on those preceding it in some ordering of the image pixels.

We propose to circumvent this difficulty by considering a given $n \times m$ image as one period of an infinite 2D signal with period $(n, m)$. Thus, at any given location $(i, j)$ in the image, the intensity values $\mathcal{I}(r, s)$ at other pixels are available also at position $(r-q n, s-q m)$, and the integer $q$ can always be chosen so that $r-q n<i, s-q m<j$. From this observation, it follows that the unknown operator $\mathcal{S}$ admits a state space representation of form (1), with the additional constraint that $\mathbf{A}^{n}=\mathbf{I}$. Finally, rather than considering a stochastic model, we will consider a deterministic equivalent:

$$
\begin{aligned}
\mathbf{x}_{k+1} & =\mathbf{A} \mathbf{x}_{k}+\mathbf{B} u_{k}, \mathbf{A}^{n}=\mathbf{I} \\
\mathbf{y}_{k} & =\mathbf{C} \mathbf{x}_{k}+\mathbf{D} u_{k} .
\end{aligned}
$$

where for each $k$, the output vector $\mathbf{y}_{k} \in R^{m}$ contains all the intensity values $\mathcal{I}(k, l), 1 \leq l \leq m$ of the pixels in the $k^{t h}$ row of the image, and where the (deterministic) input $u_{k} \in \mathcal{B S}$ captures the properties of the stochastic input process using deterministic set membership constraints [12].
Further, without loss of generality, we can assume that the specific image being considered corresponds to the case $u_{k}=\delta(0)$, by absorbing the dynamics of the input into the model, if necessary. With these assumptions, the problem becomes one of identifying a state-space realization from its impulse response data, with the additional constraint $\mathbf{A}^{n}=\mathbf{I}$, that is the dynamics are neutrally stable.

Note that this additional constraint prevents the use of currently available identification methods dealing with nonSchur plants, since most of these proceed by pre-stabilizing the unknown plant (see for instance the survey [10]), clearly not an option here. Direct identification of non-Schur dynamics was also addressed in [16], but this technique does not necessarily preserve the periodicity properties of the impulse response. On the other hand, in the specific case under consideration here, incompleteness of the information is not an issue. For texture modelling it is reasonable to assume that one has available at least one complete period of the impulse response and that these samples are noise free. Thus, a state space realization of the unknown operator can be obtained as follows:

Algorithm 2:

1.- Given an $n \times m$ image, let $\mathbf{R}_{i}^{T}$ denote its $i^{\text {th }}$ row, and form the block matrix:

$$
\mathbf{H}_{n} \doteq\left[\begin{array}{cccc}
\mathbf{R}_{2} & \mathbf{R}_{3} & \ldots & \mathbf{R}_{n} \\
\mathbf{R}_{3} & \mathbf{R}_{4} & \ldots & \mathbf{R}_{2} \\
\vdots & \vdots & \ddots & \vdots \\
\mathbf{R}_{n} & \mathbf{R}_{2} & \ldots & \mathbf{R}_{n-1}
\end{array}\right]
$$

2.- Use Algorithm 1 to obtain a balanced state-space realization.

3.- Obtain a reduced order realization by truncating the modes corresponding to the smallest $n-r$ singular values of $\mathbf{S}$.

Lemma 2: The RMS approximation error of the image when using the algorithm above is given by

$$
e=\sqrt{\sum_{\text {Image }}\left(\mathcal{I}(x, y)-\mathcal{I}_{\text {red }}(x, y)\right)^{2}}=\sqrt{\frac{\sum_{i=r+1}^{n} \sigma_{i}^{2}}{n}}
$$

where $\mathcal{I}_{\text {red }}$ denotes the image reconstructed as the impulse response of the $r^{\text {th }}$ order realization (3).

Proof: The proof follows by noting that, by construction, Trace $\left[\left(\mathbf{H}_{n}-\mathbf{H}_{n}^{\text {red }}\right)^{T}\left(\mathbf{H}_{n}-\mathbf{H}_{r}^{\text {red }}\right)\right]=n e^{2}$ and applying (5).

The procedure above is similar in spirit to a subspace identification method. The main difference is that here the special structure of the problem is exploited to explicitly obtain an exact realization (which can then be further model reduced with tight error bounds), that automatically has the required properties. In contrast, generic subspace identification methods require solving an over-determined equations system, for instance through least squares, to obtain the matrix A [18]. This (approximate) solution will not satisfy, in general, the additional constraint $\mathbf{A}^{n}=\mathbf{I}$. 


\section{B. Texture Synthesis and Image Restoration}

A partial image corresponding to one of the models above can be extended by driving its associated state space representation with a suitable input. In principle, finding this input entails searching over all candidates in $\mathcal{B S}$, since the partial image does not necessarily correspond to the impulse response of the model. However, this (infinite dimensional) search can be avoided by noting that, due to the periodicity of the image, the effect at pixel k of a (finite length) signal applied at pixel $k-s$ is the same as the effect of this signal applied at $k-s-(m * N)$, for any integer $m$, that is:

$$
\begin{aligned}
y_{k} & =\sum_{l=k-s}^{k} \mathbf{C A}^{k-l} \mathbf{B} u_{l}=\sum_{l=k-s}^{k} \mathbf{C A}^{m * N+k-l} \mathbf{B} u_{l} \\
& =\sum_{l=k-s-(m * N)} \mathbf{C A}^{k-l} \mathbf{B} \hat{u}_{l}
\end{aligned}
$$

where we have defined $\hat{u}_{l}=u_{l-m * N}$. As we show next, this observation allows for recasting the problem into a search for a suitable initial condition, which can be explicitly solved. Specifically, assuming that there is no input for $k \geq 0$, equation (7) reduces to

$$
\begin{aligned}
\mathbf{x}_{k+1} & =\mathbf{A} \mathbf{x}_{k}, \text { for some } \mathbf{x}_{o} \\
\mathbf{y}_{k} & =\mathbf{C x}_{k} .
\end{aligned}
$$

Since for each $k, \mathbf{y}_{k}$ corresponds to the $k^{t h}$ row of the image we have that $\mathcal{I}=\mathbf{K}_{o} \mathbf{x}_{o}$, where

$$
\mathbf{K}_{o}^{T}=\left[\begin{array}{llll}
\mathbf{C}^{T} & (\mathbf{C A})^{T} & \ldots & \left(\mathbf{C A}^{n-1}\right)^{T}
\end{array}\right]=\mathbf{S}^{\frac{1}{2}} \mathbf{U}^{T}
$$

Since the model is observable by construction, $\mathbf{K}_{o}$ has full column rank and the least squares solution to the equation above is given by $\mathbf{x}_{o}=\mathbf{K}_{o}^{\dagger} \mathbf{Y}$, where $\mathbf{Y}$ contains the image stacked row-wise.

The effectiveness of the proposed approach is illustrated in Table 1 showing the results of several experiments where it was used to complete the partial images shown in the first column. In all cases the model was obtained using a different portion of the image that does not overlap the swatch that was expanded.

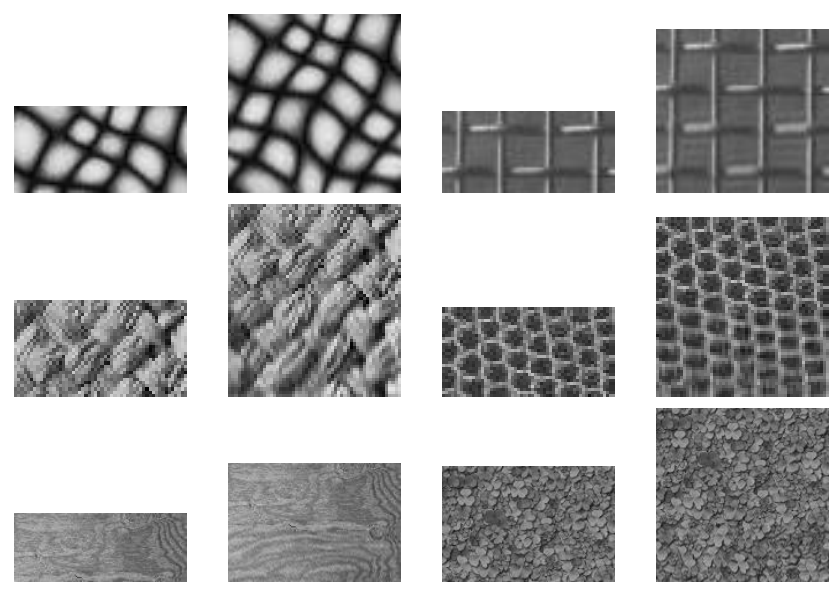

Fig. 1. Partial and Reconstructed Images

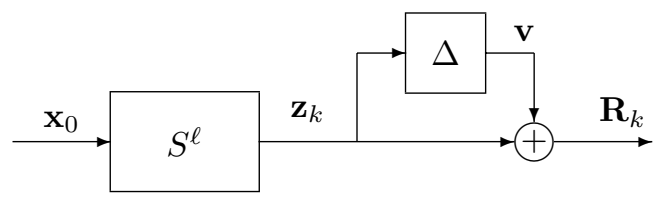

Fig. 2. The Texture Recognition Set-up

\section{Texture Recognition as a Model (In)Validation Problem}

Most texture recognition schemes rely on representations in terms of statistics of the responses to a collection of filters [6], [14]. In this paper we propose a different approach, based upon recasting the problem into a robust model (in)validation form. To this effect, we will postulate that all images corresponding to realizations of a given texture $\mathcal{T}$ can be obtained as the output of a LSI operator $S$ to an unknown input signal $e \in \mathcal{B S}$ applied in $(-\infty, 0]$, or, equivalently, as discussed in section IV-B, to an unknown initial condition $\mathbf{x}_{o}$. This leads to the set-up shown in Figure 2 , where $S^{\ell}$ represents a nominal model of a particular texture, $\mathbf{z}_{k}$ and $\mathbf{R}_{k}$ denote the rows of the ideal and actual images, respectively, and where the (unknown) operator $\Delta \in \Delta$ describes the mismatch between these two images, i.e.:

$$
\mathbf{R}_{k}=\left[(\Delta+I) S^{\ell} \mathbf{x}_{o}\right]_{k}
$$

In this framework, the texture recognition problem can be solved as follows. Given an unknown image $\mathbf{R}$ with $N<n$ rows $\mathbf{R}_{i}$ and a set of nominal models $\left\{S^{\ell}\right\}$ :

- Find for each $S^{\ell}$ an initial condition $\mathbf{x}_{0}$ and an admissible uncertainty operator $\Delta$ of minimum size $\gamma_{\text {opt }}^{\ell}$ :

$$
\gamma_{\mathrm{opt}}^{\ell} \doteq \min _{\Delta \in \Delta, x_{0}}\left\{\|\Delta\|_{*}: \mathbf{R}_{k}=\left[(\Delta+I) S^{\ell} \mathbf{x}_{0}\right]_{k}\right\} .
$$

where $\|\cdot\|_{*}$ denotes some norm of interest.

- Let $j \stackrel{\doteq}{=} \arg \min _{\ell} \gamma_{\text {opt }}^{\ell}$. Assign the image $\mathbf{R}$ to the texture represented by model $S^{j}$.

Depending on the choice of the admissible uncertainty set $\Delta$, one gets different conditions that solve (10). In the case of texture recognition, it can be argued from physical considerations that the operator $\Delta$ should be neither causal (to account for interactions amongst all pixels of the image), nor shift invariant, to allows for non-homogeneous distortions. On the other hand, linearity should be retained, to preserve invariance with respect to input scaling. Finally, we are interested in quantifying the difference between images in terms of the (relative) sum of the squared pixel errors, i.e. $\left(R_{k}-Z_{k}\right)^{T}\left(R_{k}-Z_{k}\right) /\left(Z_{k}^{T} Z_{k}\right)$. Thus, $\Delta$ should be characterized in terms of its $\ell^{2}$-induced norm. Based on these considerations, in the sequel we will assume that $\Delta \in \mathcal{B L}\left(\ell_{2}\right)(\gamma)$, and search for the smallest value of $\gamma$ so that the interconnection $(S, \Delta)$ can reproduce the given image. 


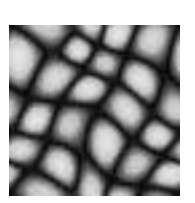

S1

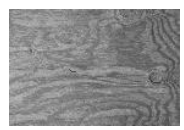

S10

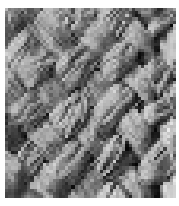

S3

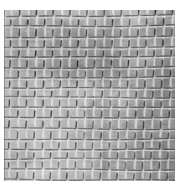

D1

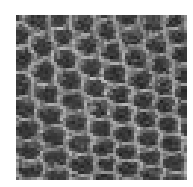

S5

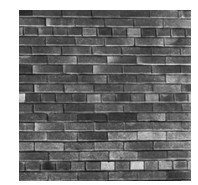

D94

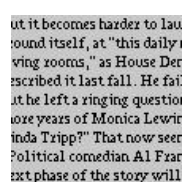

S9

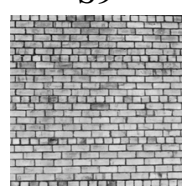

D95
Fig. 3. Sample textures used for recognition.

Recall that (see for instance Chapter 5 in [12]), given two finite vector sequences $\mathbf{v}_{k}$ and $\mathbf{z}_{\mathbf{k}}, k=0,1, \ldots n$, there exists a shift varying, not necessarily causal, operator $\Delta \epsilon$ $\mathcal{B L}\left(\ell_{2}\right)(\gamma)$ such that $\mathbf{v}=\Delta \mathbf{z}$ if and only if $\|v\|_{2}^{2} \leq \gamma^{2}\|z\|_{2}^{2}$. This result allows for recasting the search for $\Delta$ into the following LMI problem in $\mathbf{x}_{0}$ and $\alpha \doteq\left(\gamma^{2}-1\right)^{-1}$ :

$$
\begin{gathered}
\min \gamma \text { subject to : }\|v\|_{2}^{2} \leq \gamma^{2}\|z\|_{2}^{2} \\
\Longrightarrow \min \gamma \text { subject to : } \\
{\left[\begin{array}{cc}
\mathbf{R}^{T} \mathbf{R}-\mathbf{R}^{T} \mathbf{K} \mathbf{o}_{N} \mathbf{x}_{0}-\left(\mathbf{K o}_{N} \mathbf{x}_{0}\right)^{T} \mathbf{R} & \left(\mathbf{K} \mathbf{o}_{N} \mathbf{x}_{0}\right)^{T} \\
\mathbf{K o}_{N} \mathbf{x}_{0} & -\left(\gamma^{2}-1\right)^{-1}
\end{array}\right] \leq 0 .}
\end{gathered}
$$

where $\mathbf{R}$ contains the texture to be recognized, and $\mathbf{K} \mathbf{o}_{N}^{T}=$ $\left[\begin{array}{lll}\mathbf{C}^{T} & \ldots & \left(\mathbf{C A}^{N-1}\right)^{T}\end{array}\right]^{T}$

The above approach was tested on slices taken from the seven textures shown in Figure 3. To avoid biasing the models, in all cases the identification was performed using a region of the image that did not overlap the subimage to be recognized. A representative result is shown in Table I containing the minimum value of $\gamma$ obtained comparing 42 slices generated from texture $S_{10}$ against 5 candidate models. Table II shows the results of a similar experiment, where the goal is to distinguish the brick-like texture D94 from the similar looking textures D1 and D95. In order to reduce the computational complexity of the problem -given mainly by the size of the initial condition $\mathrm{x}_{0}-$ we solved (11) by estimating the initial condition $\mathrm{x}_{0}$ as:

$$
\mathbf{x}_{0}=\mathbf{K} \mathbf{o}_{n_{s}-1}^{\dagger} \mathbf{Y},
$$

where $\mathbf{K o}_{n_{s}-1}$ is the observability matrix of the nominal model under consideration and $\mathbf{Y}$ contains the given image, stacked by rows. We then computed the minimum value of $\gamma$ so that (11) was satisfied for this value of $\mathbf{x}_{0}$. As shown in both tables, in all cases this minimum indeed corresponds to the correct texture. Similar results, omitted for space reasons, were obtained with all textures. Overall, in more than 400 experiments we achieved a recognition rate of over $98 \%$.

\begin{tabular}{|c|c|c|c|c|c|}
\hline Image & $S_{1}$ & $S_{3}$ & $S_{5}$ & $S_{9}$ & $S_{10}$ \\
\hline 1 & 0.1282 & 0.1573 & 0.1251 & 0.1543 & 0.0715 \\
2 & 0.1287 & 0.1583 & 0.1255 & 0.1554 & 0.0722 \\
3 & 0.1293 & 0.1594 & 0.1262 & 0.1556 & 0.0735 \\
4 & 0.1294 & 0.1594 & 0.1260 & 0.1557 & 0.0759 \\
5 & 0.1410 & 0.1601 & 0.1264 & 0.1562 & 0.0790 \\
6 & 0.1299 & 0.1604 & 0.1260 & 0.1563 & 0.0789 \\
7 & 0.1303 & 0.1610 & 0.1264 & 0.1564 & 0.0799 \\
8 & 0.1314 & 0.1611 & 0.1274 & 0.1567 & 0.0828 \\
9 & 0.1318 & 0.1608 & 0.1272 & 0.1570 & 0.0668 \\
10 & 0.1317 & 0.1609 & 0.1274 & 0.1574 & 0.0673 \\
11 & 0.1316 & 0.1616 & 0.1279 & 0.1633 & 0.0675 \\
12 & 0.1313 & 0.1609 & 0.1280 & 0.1676 & 0.0678 \\
13 & 0.1348 & 0.1603 & 0.1316 & 0.1743 & 0.0680 \\
14 & 0.1318 & 0.1600 & 0.1284 & 0.1734 & 0.0683 \\
15 & 0.1318 & 0.1618 & 0.1289 & 0.1573 & 0.0685 \\
16 & 0.1317 & 0.1629 & 0.1298 & 0.1575 & 0.0688 \\
17 & 0.1319 & 0.1625 & 0.1300 & 0.1580 & 0.0689 \\
18 & 0.1324 & 0.1625 & 0.1305 & 0.1585 & 0.0691 \\
19 & 0.1337 & 0.1633 & 0.1313 & 0.1587 & 0.0693 \\
20 & 0.1347 & 0.1637 & 0.1317 & 0.1591 & 0.0696 \\
21 & 0.1341 & 0.1648 & 0.1318 & 0.1595 & 0.0698 \\
22 & 0.1340 & 0.1641 & 0.1324 & 0.1634 & 0.0701 \\
23 & 0.1342 & 0.1638 & 0.1330 & 0.1602 & 0.0704 \\
24 & 0.1346 & 0.1623 & 0.1337 & 0.1610 & 0.0707 \\
25 & 0.1350 & 0.1618 & 0.1337 & 0.1624 & 0.0709 \\
26 & 0.1352 & 0.1609 & 0.1341 & 0.1653 & 0.0711 \\
27 & 0.1356 & 0.1605 & 0.1346 & 0.1668 & 0.0712 \\
28 & 0.1398 & 0.1608 & 0.1357 & 0.1707 & 0.0714 \\
29 & 0.1366 & 0.1613 & 0.1359 & 0.1698 & 0.0714 \\
30 & 0.1406 & 0.1629 & 0.1409 & 0.1750 & 0.0718 \\
31 & 0.1505 & 0.1643 & 0.1448 & 0.1821 & 0.0720 \\
32 & 0.1584 & 0.1711 & 0.1522 & 0.1967 & 0.0720 \\
33 & 0.1713 & 0.1781 & 0.1566 & 0.1883 & 0.0722 \\
34 & 0.1840 & 0.1800 & 0.1593 & 0.1924 & 0.0726 \\
35 & 0.1457 & 0.1602 & 0.1553 & 0.1638 & 0.0729 \\
36 & 0.1447 & 0.1591 & 0.1388 & 0.1602 & 0.0732 \\
37 & 0.1312 & 0.1605 & 0.1344 & 0.1583 & 0.0733 \\
38 & 0.1321 & 0.1619 & 0.1358 & 0.1586 & 0.0735 \\
39 & 0.1346 & 0.1638 & 0.1372 & 0.1589 & 0.0740 \\
40 & 0.1472 & 0.1656 & 0.1388 & 0.1595 & 0.0747 \\
41 & 0.1329 & 0.1660 & 0.1389 & 0.1602 & 0.0749 \\
42 & 0.1346 & 0.1653 & 0.1401 & 0.1610 & 0.0755 \\
\hline
\end{tabular}

TABLE I

RESULTS FOR TEXTURE 10

\section{Conclusions}

Many problems of practical interest require addressing the issues of identification and model reduction of systems having a periodic impulse response. Currently available techniques are not well suited for solving these problems, since they cannot guarantee that key structural properties, such as periodicity of the impulse response, will be preserved.

Motivated by existing subspace identification methods and their relationship with well known results in realization theory, in this paper we address these problems by working directly with the Hankel matrix of the system under consideration. The main result of the paper shows that, due to the block circulant structure, a direct SVD-based 


\begin{tabular}{|c|c|c|c|}
\hline Image & $S_{\text {brick }}^{1}$ & $S_{\text {brick }}^{94}$ & $S_{\text {brick }}^{95}$ \\
\hline 1 & 0.4349 & 0.1956 & 0.4873 \\
2 & 0.4589 & 0.3605 & 0.4586 \\
3 & 0.4720 & 0.4545 & 0.4972 \\
4 & 0.5599 & 0.5075 & 0.5154 \\
\hline
\end{tabular}

TABLE II

RESUlTS FOR BRICK TEXTURE 94

approximation of this matrix also has a Hankel structure, and that this procedure is equivalent to performing balanced truncations based on the finite-time grammians.

These results are illustrated with a non-trivial practical example arising in the context of image processing: texture synthesis and recognition. Here the proposed method leads to low order, parsimonious models capable of generating images with the desired texture when excited with appropriate initial conditions (or equivalently, with signals with unit spectral density). Moreover, these models can be successfully used to both restore partial images and to classify an unknown sample. A potential drawback of the proposed technique is that at this stage it requires ordering the pixels of the image either row or column-wise, leading to potentially different models if the image exhibits substantially different behavior in these two directions. Efforts are currently under way to remove this limitation by extending the proposed approach to 2-D systems.

\section{REFERENCES}

[1] V. M. Adamjan, D. Z. Arov, and M. G. Krein, "Infinite Hankel block matrices and related extension problems," AMS Translations, vol. $111,1978$.

[2] U. M. Al-Saggaf, On Model Reduction and Control of Discrete Time Systems. Ph.D. Thesis, Stanford University, 1986.

[3] J. Chen and G. Gu, Control Oriented System Identification, An $\mathcal{H}_{\infty}$ Approach. New York: John Wiley, 2000.

[4] A. Doherty, M. Barahona, J. Doyle, H. Mabuchi, and M. Sznaier, "Robustness and dynamics in the quantum classical transition," in Sixth SIAM Conference on Applications of Dynamical Systems, Snowbird, UT, May 2001.

[5] A. Efros and T. Leung, "Texture synthesis by non-parametric sampling," in ICCV, 1999.

[6] D. Forsyth and J. Ponce, Computer Vision: A Modern Approach. Prentice Hall, 2003.

[7] K. Glover, "All optimal Hankel norm approximations of linear multivariable systems and their $\mathcal{L}_{\infty}$ error bounds," Int. J. of Control, vol. 39, no. 6, pp. 1115-1193, 1994.

[8] M. H. Khammash and J. B. Pearson, "Performance robustness of discrete-time systems with structured uncertainty," IEEE Transactions on Automatic Control, vol. 36, no. 4, 1991.

[9] S. K. Kung, "A new low order approximation algorithm via singular value decomposition," in $12^{\text {th }}$ Asilomar Conf. Circ. Syst. and Comp., 1978, pp. 705-714.

[10] P. M. Mäkilä, J. R. Partington, and T. K. Gustafsson, "Worstcase control-relevant identification," Automatica, vol. 31, no. 12, pp. 1799-1819, 1995.

[11] B. C. Moore, "Principal component analysis in linear systems: Controllability, observability and model reduction," IEEE Trans. Aut. Control, vol. 26, no. 1, pp. 17-32, 1981.

[12] F. Paganini, Sets and Constraints in the Analysis of Uncertain Systems. Ph. D. Dissertation, California Institute of Technology, 1996.

[13] R. Sánchez Peña and M. Sznaier, Robust Systems Theory and Applications. Wiley \& Sons, Inc., 1998.
[14] M. Sonka, V. Hlavac, and R. Boyle, Image Processing, Analysis, and Machine Vision. PWS Publishing, 1999.

[15] M. Sznaier, A. Doherty, M. Barahona, H. Mabuchi, and J. Doyle, "A new bound of the $\mathcal{L}_{2}$ induced norm and applications to model reduction," in 2002 American Control Conference, Anchorage, Alaska, May 2002.

[16] M. Sznaier, C. Mazzaro, and O. I. Camps, "Open-loop worst-case identification of nonschur plants," Automatica, vol. 39, no. 6, pp. 1019-1025, June 2003.

[17] A. J. Tether, "Construction of minimal linear state variable models from finite input/output data," IEEE Trans. Aut. Control, vol. 15, pp. 427-436, 1981

[18] P. Van Overschee and B. De Moor, "Subspace algorithms for the stochastic identification problem," Automatica, vol. 29 , no. 3, pp. 649-660, May 1993.

[19] H. P. Zeiger and A. J. McEwen, "Approximate linear realizations of given dimension via ho's algorithm," IEEE Trans. Aut. Control, vol. 19, p. 153, 1974.

[20] K. Zhou, J. C. Doyle, and K. Glover, Robust and Optimal Control. Prentice Hall, 1996.

[21] K. Zhou, G. Salomon, and E. Wu, "Balanced realization and model reduction for unstable systems," Int. J. Robust and Nonlinear Ctrl., vol. 9, pp. 183-189, 1999.

\section{APPENDIX}

\section{A. Proof of Lemma 1}

Start by partitioning $\mathbf{U}=\left[\begin{array}{ll}\mathbf{U}_{r} & \mathbf{U}_{n-r}\end{array}\right]$. Since $\mathbf{P} \mathbf{U}_{r}$ is a circular block permutation of $\mathbf{U}_{r}$, then $\mathbf{U}_{r} \mathbf{U}_{r}^{T} \mathbf{P} \mathbf{U}_{r}=$ $\left(\mathbf{I}-\mathbf{U}_{n-r} \mathbf{U}_{n-r}^{T}-\mathbf{U}_{\perp} \mathbf{U}_{\perp}^{T}\right) \mathbf{P} \mathbf{U}_{r}=\mathbf{P} \mathbf{U}_{r}$. It follows that $\mathbf{A}_{r}^{k}=\mathbf{S}_{r}^{\frac{-1}{2}} \mathbf{U}_{r}^{T} \mathbf{P}^{k} \mathbf{U}_{r} \mathbf{S}_{r}^{\frac{1}{2}}$. The fact that $\mathbf{A}^{n}=I$ follows directly from $\mathbf{P}^{n}=I$. Straightforward computations (omitted due to space constraints) using the expressions for $\mathbf{C}_{r}, \mathbf{B}_{r}$ and $\mathbf{A}_{r}^{k}$ yield

$$
\begin{aligned}
& \mathbf{M}_{k}^{r e d} \doteq \mathbf{C}_{r} \mathbf{A}_{r}^{k-1} \mathbf{B}_{r}=\mathbf{E}^{(1)} \mathbf{P}^{(k-1)} \mathbf{U}_{r} \mathbf{S}_{r} \mathbf{V}_{r}^{(1)} \\
& \mathbf{E}^{(1)} \doteq\left[\mathbf{I}_{p} \ldots \mathbf{0} \ldots \mathbf{0}\right]
\end{aligned}
$$

From the (block) symmetry properties of $\mathbf{H}_{n}$ it follows that for any $k, j, \mathbf{U}^{(k)} \mathbf{S} \mathbf{V}^{(l)}=\mathbf{U}^{(l)} \mathbf{S} \mathbf{V}^{(j)}$, where $\mathbf{U}^{(k)}$ and $\mathbf{V}^{(j)}$ denote the $k^{\text {th }}$ and $j^{\text {th }}$ blocks of $\mathbf{U}$ and $\mathbf{V}$, respectively. Combining this observation with (13) and the fact that

$$
\mathbf{E}^{(1)} \mathbf{P}^{(k)}=\mathbf{E}^{(k)} \doteq[\underbrace{\mathbf{0} \ldots \mathbf{0}}_{k-1} \mathbf{I}_{p} \ldots \mathbf{0}]
$$

yields $\mathbf{H}_{n}^{r e d}=\mathbf{U}_{r} \mathbf{S}_{r} \mathbf{V}_{r}$. Hence,

$$
\mathbf{H}_{n}-\mathbf{H}_{n}^{r e d}=\mathbf{U}_{H}\left[\begin{array}{lll}
0 & & \\
& \mathbf{S}_{n-r} & \\
& & 0
\end{array}\right] \mathbf{V}_{H}^{T}
$$

where $\mathbf{U}_{H}=\left[\begin{array}{ll}\mathbf{U}_{r} & \mathbf{U}_{n-r} \mathbf{U}_{\perp}\end{array}\right]$ and $\mathbf{V}_{H}=$ $\left[\begin{array}{ll}\mathbf{V}_{r} & \mathbf{V}_{n-r} \mathbf{V}_{\perp}\end{array}\right]$. The bound (5) follows now immediately from the fact that $\mathbf{U}_{H}$ and $\mathbf{V}_{H}$ are unitary. 